\title{
Floquet Majorana bound states in voltage-biased planar Josephson junctions
}

\author{
Changnan Peng $\odot,{ }^{1,2}$ Arbel Haim,,${ }^{1,3,4}$ Torsten Karzig $\odot,{ }^{5}$ Yang Peng, ${ }^{6,1,3}$ and Gil Refael ${ }^{1,3}$ \\ ${ }^{1}$ Institute for Quantum Information and Matter and Department of Physics, California Institute of Technology, \\ Pasadena, California 91125, USA \\ ${ }^{2}$ Department of Physics, Massachusetts Institute of Technology, Cambridge, Massachusetts 02139, USA \\ ${ }^{3}$ Walter Burke Institute for Theoretical Physics, California Institute of Technology, Pasadena, California 91125, USA \\ ${ }^{4}$ AWS Center for Quantum Computing, Pasadena, California 91125, USA \\ ${ }^{5}$ Microsoft Quantum, Station Q, University of California, Santa Barbara, California 93106, USA \\ ${ }^{6}$ Department of Physics and Astronomy, California State University, Northridge, California 91330, USA
}

(Received 11 January 2021; revised 11 April 2021; accepted 13 April 2021; published 10 May 2021)

\begin{abstract}
We study a planar Josephson junction under an applied DC voltage bias in the presence of an in-plane magnetic field. Upon tuning the bias voltage across the junction $V_{\mathrm{J}}$, the two ends of the junction are shown to simultaneously host both zero and $\pi$ Majorana modes. These modes can be probed using either a scanningtunneling-microscopy measurement or through resonant Andreev tunneling from a lead coupled to the junction. While these modes are mostly bound to the junction's ends, they can hybridize with the bulk by absorbing or emitting photons. We analyze this process both numerically and analytically, demonstrating that it can become negligible under typical experimental conditions. Transport signatures of the zero and $\pi$ Majorana states are shown to be robust to moderate disorder.
\end{abstract}

DOI: 10.1103/PhysRevResearch.3.023108

\section{INTRODUCTION}

For some time, the idea of Floquet Majoranas has been an intriguing concept that has brought together the fields of nonAbelian anyons, quantum computing, and quantum dynamics. Floquet Majorana states were first proposed in Ref. [1] and since have been the focus of much discussion. The most direct impact that Floquet Majoranas had was conceptual. Being excitations that are pinned to the quasienergy which is half the drive frequency, the Floquet Majoranas are the archetype of the time crystal phenomenon [2-6].

The study of Majoranas in driven systems is also motivated by the need to expand our control tools of quantum information processing elements. A drive can also enhance the functionality of standard platforms for non-Abelian excitations. Recently, it was recognized as a way to expand the effective dimensionality of a Majorana system, allowing braiding of Majoranas even in a strict one-dimensional (1D) wire system [7-9]. Also, by using the drive-induced synthetic dimensions concept [10-12], one can use drives to expand the number of non-Abelian anyons that can be supported on a single Majorana wire (see, for instance, Ref. [13]).

How does one best realize Floquet Majorana states experimentally? Reference [14] proposed a realization that is in line with the original proposal in Ref. [1]. This proposal involves

Published by the American Physical Society under the terms of the Creative Commons Attribution 4.0 International license. Further distribution of this work must maintain attribution to the author(s) and the published article's title, journal citation, and DOI. the oscillation of the gate voltage applied to the system to produce the time dependence needed. Other proposals include driven quantum dots [15] and quantum wires [16-21]. In this paper we show that Floquet Majoranas can emerge even in much simpler systems that are currently experimentally available. Particularly, we analyze a spin-orbit coupled strip, placed in proximity to a superconductor on each of its sides, as considered in Refs. [22-32].

As we show below, this system naturally gives rise to two sets of Majorana end states when a DC bias is maintained between the two superconductors. Indeed, through the AC Josephson effect, such a system appears to be driven by the Josephson frequency $\Omega=2 e V_{\mathrm{J}} / \hbar$. We demonstrate the appearance of Floquet Majoranas using numerical simulations, as well as analytical arguments. We also explore the robustness of the Floquet Majoranas to disorder.

The rest of this paper is organized as follows. We begin in Sec. II by describing the system and explain how it can give rise to a Floquet type of topological superconductivity which gives rise to zero and $\pi$ Majorana states. We then perform numerical transport simulations in Sec. III, demonstrating their existence in a way similar to how they should manifest in experiment. In Sec. IV we analyze the effect of photonmediated coupling between Majorana states on opposite sides of the system. We conclude and discuss future prospects in Sec. V.

\section{THE SYSTEM}

We consider a Josephson junction, constructed by proximitizing two conventional superconductors to a two-dimensional electron gas (2DEG) with an in-plane applied magnetic field. 
(a)

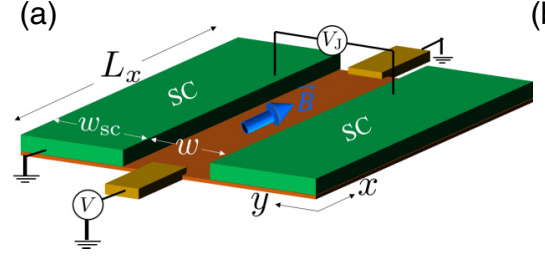

(b)

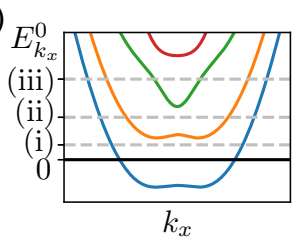

FIG. 1. (a) Proposed experimental setup. A voltage-biased planar Josephson junction implemented in a Rashba spin-orbit coupled 2DEG in the presence of an in plane-magnetic field. (b) Low-energy spectrum of the 2DEG strip in the absence of superconductivity. The Fermi level $(\varepsilon=0)$ is marked by a solid black line, and $\varepsilon=\Omega / 2$ is marked by a gray dashed line, where three different scenarios are considered. A $\pi$ Majorana state emerges at the junction ends whenever $\Omega / 2$ crosses an odd number of bands. The scenarios (i) $V_{\mathrm{J}}=43.75 \mu \mathrm{V}$, (ii) $V_{\mathrm{J}}=125 \mu \mathrm{V}$, and (iii) $V_{\mathrm{J}}=237.5 \mu \mathrm{V}$ correspond to Figs. 2(a)-2(f), respectively. The parameters used to obtain the spectrum are the same as those given in the text, i.e., $E_{\mathrm{so}}=100 \mu \mathrm{eV}, E_{\mathrm{Z}}^{0}=75 \mu \mathrm{eV}$, etc. (see the end of Sec. II), except that here the chemical potential is shifted to $\mu_{\mathrm{J}}=0 \mu \mathrm{eV}$ in order to compensate for the absence of the superconductors.

Importantly, a DC bias voltage $V_{\mathrm{J}}$ is applied across the junction between the two superconductors. To probe the system using electric transport, we further consider two normal-metal leads coupled to the two ends of the junction. The system is depicted in Fig. 1(a).

The Hamiltonian describing this system in the absence of the normal-metallic leads is described by

$$
\begin{aligned}
\mathcal{H}(t)= & {\left[-\frac{\nabla^{2}}{2 m_{\mathrm{e}}}-\mu(y)+U(x, y)-i \alpha\left(\sigma_{y} \partial_{x}-\sigma_{x} \partial_{y}\right)\right] \tau_{z} } \\
& +E_{\mathrm{Z}}(y) \sigma_{x}+\operatorname{Re}[\Delta(y)] \tau_{x}-\operatorname{Im}[\Delta(y)] \tau_{y} .
\end{aligned}
$$

This Hamiltonian is written in the basis of the Nambu spinor $\Psi^{\dagger}(\boldsymbol{r})=\left[\psi_{\uparrow}^{\dagger}(\boldsymbol{r}), \psi_{\downarrow}^{\dagger}(\boldsymbol{r}), \psi_{\downarrow}(\boldsymbol{r}),-\psi_{\uparrow}(\boldsymbol{r})\right]$, where $\psi_{s}^{\dagger}(\boldsymbol{r})$ creates an electron inside the 2DEG at position $\boldsymbol{r}=(x, y)$ with spin $s$. The Pauli matrices $\left\{\sigma_{x, y, z}\right\}$ and $\left\{\tau_{x, y, z}\right\}$ operate on the spin and particle-hole degrees of freedom, respectively. Here, $m_{\mathrm{e}}$ is the effective electron mass in the 2DEG; $\mu(y)=\mu_{\mathrm{J}} \Theta(w / 2-$ $|y|)+\mu_{\mathrm{SC}} \Theta(|y|-w / 2)$ is the chemical potential, where $\mu_{\mathrm{J}}$ $\left(\mu_{\mathrm{SC}}\right)$ is its value in the junction (below the superconductors), $\Theta$ is the Heaviside step function, and $y=0$ denotes the middle of the system; $U(x, y)$ is a disorder potential due to impurities; $\alpha$ is the Rashba spin-orbit coupling coefficient; and $E_{Z}(y)=E_{Z}^{0} \Theta(w / 2-|y|)$ is the Zeeman splitting due to the in-plane magnetic field present in the junction. Finally, the induced superconducting potential inside the $2 \mathrm{DEG}$ is given by $\Delta(y)=\Delta_{0} \Theta(|y|-w / 2) \exp [i \Theta(y) \phi(t)]$, where a linearly time-dependent phase bias $\phi(t)=2 e V_{\mathrm{J}} t$ is generated by the voltage across the junction.

As a result of the oscillating phase between the superconductors, the Hamiltonian of Eq. (1) is time periodic, $\mathcal{H}(t+$ $T)=\mathcal{H}(t)$, with a period $T=\pi /\left(e V_{\mathrm{J}}\right)$. We can accordingly write the Hamiltonian using its Floquet representation,

$$
\mathcal{H}_{m n}^{\mathrm{F}}=n \Omega \delta_{m n}+\frac{1}{T} \int_{0}^{T} d t e^{-i(m-n) \Omega t} \mathcal{H}(t),
$$

where $\Omega=2 \pi / T=2 e V_{\mathrm{J}}$ and $m, n \in \mathbb{Z}$. By construction, the spectrum of $\mathcal{H}^{\mathrm{F}}$ is periodic under $\varepsilon \rightarrow \varepsilon+\Omega$. The Flo- quet Hamiltonian further obeys a particle-hole symmetry, $\tau_{y} \sigma_{y}\left[\mathcal{H}^{\mathrm{F}}{ }_{m, n}\right]^{*} \tau_{y} \sigma_{y}=-\mathcal{H}^{\mathrm{F}}{ }_{-m,-n}$, dictating a symmetry of the spectrum under $\varepsilon \rightarrow-\varepsilon$. Together with the periodicity of the spectrum, one concludes that a single state with either $\varepsilon=0$ or $\varepsilon=\Omega / 2$ is protected and cannot be removed by any perturbation respecting these symmetries [1]. Such states are referred to as zero Majorana and $\pi$ Majorana states, respectively, where zero and $\pi$ correspond to the phase acquired by these states upon a unitary evolution over a time $T$.

To gain some intuition, one can first consider the weakpairing limit. In this limit the induced superconducting pairing inside the junction can be treated as a small perturbation to the band structure of an isolated semiconducting strip. This band structure, shown in Fig. 1(b), contains multiple transverse bands which are spin split due to spin-orbit coupling and magnetic field. As in the stationary case of a topological superconductor [33-37], one expects zero Majorana states to emerge when the Fermi level (black solid line), $\varepsilon=0$, crosses an odd number of bands (namely, an odd number of pairs of Fermi points). In the case of a driven (Floquet) topological superconductor, one expects, in addition, $\pi$ Majorana states to emerge whenever the line $\varepsilon=\Omega / 2$ (gray dashed line) crosses through an odd number of bands. Below, we consider three different values of $V_{\mathrm{J}}$ corresponding to $\varepsilon=\Omega / 2$ crossing either one, two, or three bands.

Throughout this work, we take the system parameters to be $\Delta_{0}=500 \mu \mathrm{eV}, E_{\mathrm{so}}=m_{\mathrm{e}} \alpha^{2} / 2=100 \mu \mathrm{eV}, l_{\mathrm{so}}=\hbar /\left(m_{\mathrm{e}} \alpha\right)=$ $100 \mathrm{~nm}, \mu_{\mathrm{J}}=37.5 \mu \mathrm{eV}, \mu_{\mathrm{SC}}=1 \mathrm{meV}, E_{\mathrm{Z}}^{0}=75 \mu \mathrm{eV}, w=$ $292 \mathrm{~nm}$, and $w_{\mathrm{sc}}=292 \mathrm{~nm}$. This set of parameters corresponds to $m_{\mathrm{e}}=3.47 \times 10^{-32} \mathrm{~kg}=0.038 m_{\mathrm{e}}^{0}$ and $\alpha=3.04 \times$ $10^{4} \mathrm{~m} / \mathrm{s}$.

\section{NUMERICAL ANALYSIS}

To simulate the system numerically, we truncate the Floquet indices $m, n \in\left[-N_{\mathrm{F}}, N_{\mathrm{F}}\right]$ in Eq. (2). For a large enough cutoff $N_{\mathrm{F}}$ this is justified by the frequency-space localization of the Floquet eigenstates, which is induced by the term $n \Omega \delta_{m n}$. We further discretize the Hamiltonian $\mathcal{H}_{m n}^{\mathrm{F}}$ spatially by constructing an appropriate tight-binding Hamiltonian on a rectangular lattice. In the present work we keep seven Floquet bands $\left(N_{\mathrm{F}}=3\right)$ and take the lattice constants to be $a_{x}=40 \mathrm{~nm}$ and $a_{y}=73 \mathrm{~nm}$.

To probe the presence of Majorana modes we consider the case where two normal-metallic leads are connected to the two ends of the junction, as depicted in Fig. 1(a). In this setup, the presence of zero and $\pi$ Majorana states at the junction ends will induce resonant Andreev reflection of an electron arriving from one of the normal-metal leads with energy zero and $e V_{\mathrm{J}}$, respectively. Experimentally, this should be observed in the DC differential conductance, $\sigma(V)=d I_{\mathrm{DC}} / d V$, where $I_{\mathrm{DC}}$ is the DC component of the current in the normal-metal lead and $V$ is its voltage with respect to ground.

To obtain this quantity numerically, we calculate the scattering matrix of the discretized truncated Floquet Hamiltonian, with the reflection and transmission blocks having the form

$$
r_{i m ; j n}=\left(\begin{array}{cc}
r_{i m ; j n}^{\mathrm{ee}} & r_{i m ; j n}^{\mathrm{eh}} \\
r_{i m ; j n}^{\mathrm{he}} & r_{i m ; j n}^{\mathrm{hh}}
\end{array}\right), \quad t_{i m ; j n}=\left(\begin{array}{cc}
t_{i m ; j n}^{\mathrm{ee}} & t_{i m ; j n}^{\mathrm{eh}} \\
t_{i m ; j n}^{\mathrm{he}} & t_{i m ; j n}^{\mathrm{hh}}
\end{array}\right) .
$$


(a)

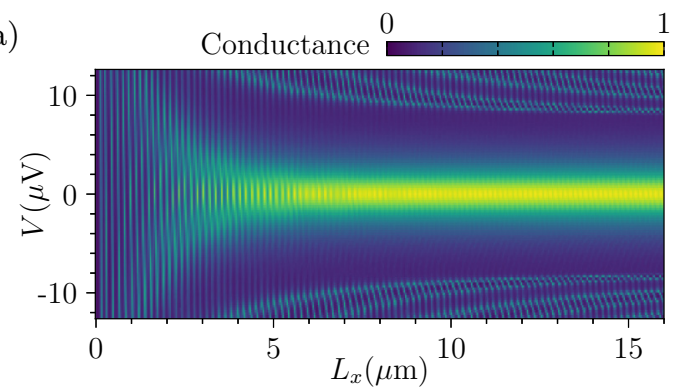

(c)

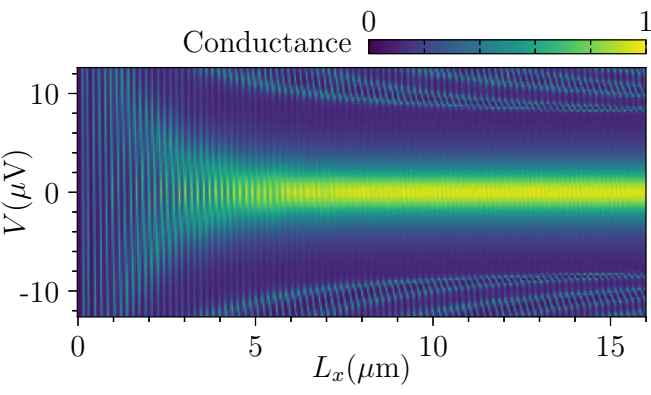

(e)

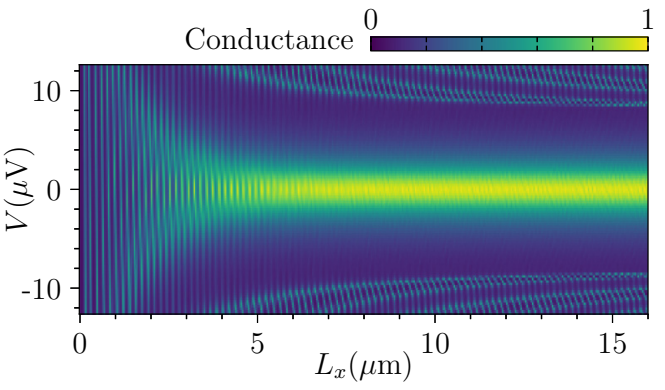

(b)

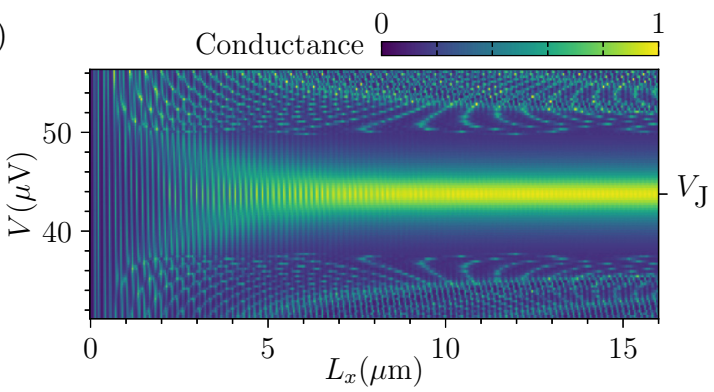

(d)
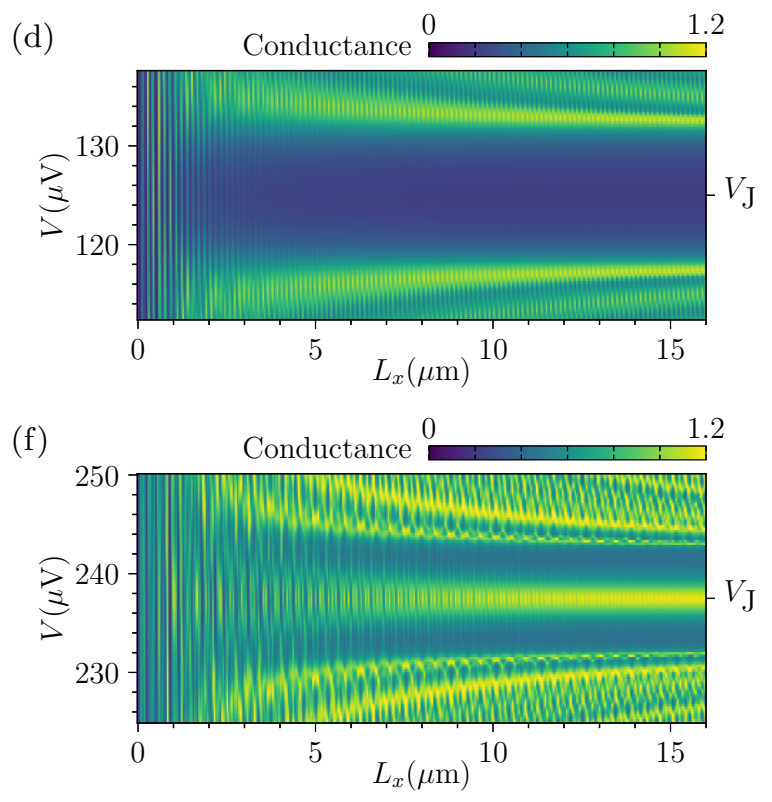

FIG. 2. Conductance as a function of the lead's voltage $V$ and the junction's length $L_{x}$, in units of $2 e^{2} / h$. The voltage across the junction is taken to be (a) and (b) $V_{\mathrm{J}}=43.75 \mu \mathrm{V}$, (c) and (d) $V_{\mathrm{J}}=125 \mu \mathrm{V}$, and (e) and (f) $V_{\mathrm{J}}=237.5 \mu \mathrm{V}$. These three values correspond to the three dashed gray lines in Fig. 1(b), marked (i), (ii), and (iii), respectively.

For example, $r_{i m ; j n}^{\text {he }} \equiv r_{i j}^{\text {he }}(\varepsilon+m \Omega, \varepsilon+n \Omega)$ is the amplitude for an electron in mode $(i, m)$ to be reflected as a hole in mode $(j, n)$, where $m, n$ label the Floquet sectors and $i, j$ each label the spin and transverse modes in the lead. The scattering matrix is calculated using the recursive Green's function technique [38] (see Refs. [29,39] for details of implementation).

The DC differential conductance can then be extracted from the scattering matrix using the Landauer-Büttiker formalism, generalized for a periodically driven superconducting system,

$$
\begin{aligned}
\sigma(V)= & \frac{e^{2}}{h} \sum_{i j} \sum_{n=-\infty}^{\infty}\left[\left|t_{i j}^{\mathrm{ee}}(e V, e V+n \Omega)\right|^{2}\right. \\
& \left.+\left|t_{i j}^{\mathrm{he}}(e V, e V+n \Omega)\right|^{2}+2\left|r_{i j}^{\mathrm{he}}(e V, e V+n \Omega)\right|^{2}\right] .
\end{aligned}
$$

Terms involving $t^{\mathrm{ee}}$ and $t^{\mathrm{he}}$ describe processes where a single electron is emitted, therefore contributing a unit quantum conductance, while the Andreev reflection term $r^{\text {he }}$ describes a process where two electrons are emitted from the lead and therefore contribute two units of quantum conductance [40]. Unlike the case of a stationary system, however, each of these processes can now occur through an absorption or emission of $n$ photons [41-43].
In Fig. 2, we present results for $\sigma(V)=d I_{\mathrm{DC}} / d V$ as a function of the junction's length $L_{x}$ [see Fig. 1(a)] and the voltage in the lead $V$ for a clean system. Figures 2(a), 2(c), and 2(e) focus on voltages near $V=0$, while Figs. 2(b), 2(d), and 2(f) focus on voltages near $V=V_{\mathrm{J}}$. For a long enough system, the emergence of zero and/or $\pi$ Majorana states can be seen as a robust resonance at $V=0$ and/or $V=V_{\mathrm{J}}$, respectively.

The top, middle, and bottom panels correspond to three different values of the voltage $V_{\mathrm{J}}$ across the junction. These three values of $V_{\mathrm{J}}$ are shown in Fig. 1(b) as gray dashed lines labeled (i), (ii), and (iii). They are chosen such that $\varepsilon=\Omega / 2=e V_{\mathrm{J}}$ crosses either a single band [Figs. 2(a) and 2(b)], two bands [Fig. 2(c) and 2(d)], or three bands [Fig. 2(e) and 2(f)]. As expected, $\pi$ Majorana modes emerge when the number of bands crossed by $\varepsilon=\Omega / 2$ is odd. In all these cases the chemical potential $\mu$ is kept constant with the Fermi level $(\varepsilon=0)$ crossing a single band, as shown in Fig. 1(b). Signatures of zero Majorana states can accordingly be seen in all the left panels of Fig. 2.

In the stationary case of a topological superconductor, under some general conditions the conductance resonance is quantized to $\sigma(0)=2 e^{2} / h$ at zero temperature [44-47]. More specifically, if the system is gapped and long enough, the transmission matrix vanishes, and the reflection matrix can be shown to have a single perfectly-Andreev-reflecting channel 


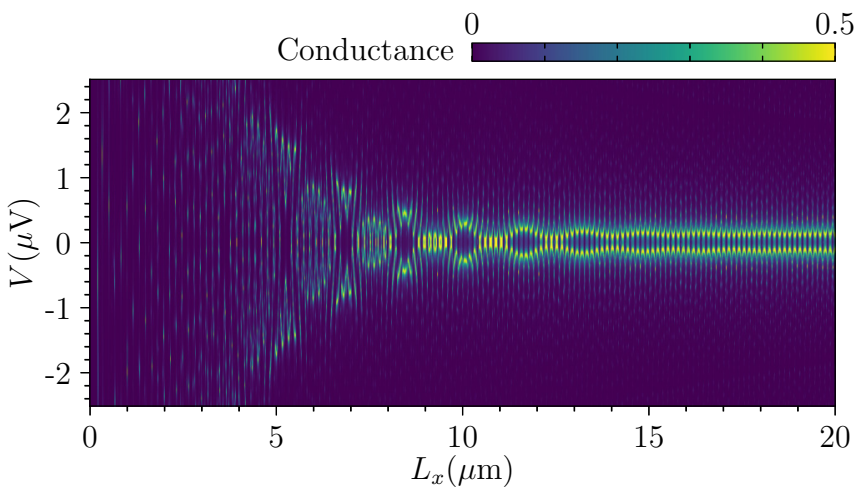

FIG. 3. Conductance as a function of the lead's voltage $V$ and the junction's length $L_{x}$, in units of $2 e^{2} / h$. System parameters are the same as in Figs. 2(a) and 2(b), except the coupling to the lead is reduced, enabling us to view the splitting of the Majorana resonance. The splitting does not decrease to zero with increasing $L_{x}$ and is most likely a result of photon-mediated coupling between Majorana states on opposite ends of the junction (see Sec. IV).

in the topological phase [48]. If, in addition, the lead is weakly coupled to the system (or if the lead has a single channel), the rest of the channels do not contribute, yielding $\sigma(0)=2 e^{2} / h$.

In contrast, for a periodically driven topological superconductor the resonances at $V=0$ and $V=V_{\mathrm{J}}$ are generally not quantized, even in the limit of a weakly coupled lead and a gapped infinite system. Instead, quantization is obtained only when summing over the differential conductance at certain discrete energies $[43,49]$. In the presence of a zero Majorana bound state one has $\sum_{m} \sigma\left(2 m e V_{\mathrm{J}}\right)=2 e^{2} / h$, while in the presence of a $\pi$ Majorana bound state one has $\sum_{m} \sigma[(2 m+$ 1) $\left.e V_{\mathrm{J}}\right]=2 e^{2} / h$.

The resonances seen in Figs. 2(a)-2(c) and 2(e) exhibit a peak value only slightly less than $2 e^{2} / h$. This can suggest that conductances at $V=2 m e V_{\mathrm{J}}$ and $V=(2 m+1) e V_{\mathrm{J}}$ with $m \neq$ 0 are relatively suppressed. This is reasonable considering that electrons arriving at these energies require the absorption or emission of several photons in order be in resonance with the Majorana states. For $\left|\Delta_{\text {ind }}\right|<|\Omega|$, which is the case considered here, such processes would be suppressed. In the case of Fig. 2(f), on the other hand, the conductance resonance exhibits a peak value slightly above $2 e^{2} / h$. This is a result of the coupling to the lead being comparable with the induced gap, allowing for higher-energy states to contribute to conductance. Indeed, the induced gap around $V_{\mathrm{J}}$ shown in Fig. 2(f) is smaller than the gaps seen in the spectra of Figs. 2(a)-2(e).

To examine the Majorana-induced resonance with better resolution, we consider the conductance for the case shown in Fig. 2(a), but with a weaker coupling between the system and the normal-metal leads. This weaker coupling causes the width of the resonance to decrease, allowing for a closer examination of the splitting of the Majorana modes. The result is shown in Fig. 3. One can now clearly observe the splitting of the resonance away from $V=0$. As the junction's length $L_{x}$ is increased, the resonance energy initially oscillates with a decreasing amplitude; however, beyond about $L_{x} \sim 10 \mu \mathrm{m}$ the splitting approaches a constant value. This behavior is quite different from that of a static topological superconductor,
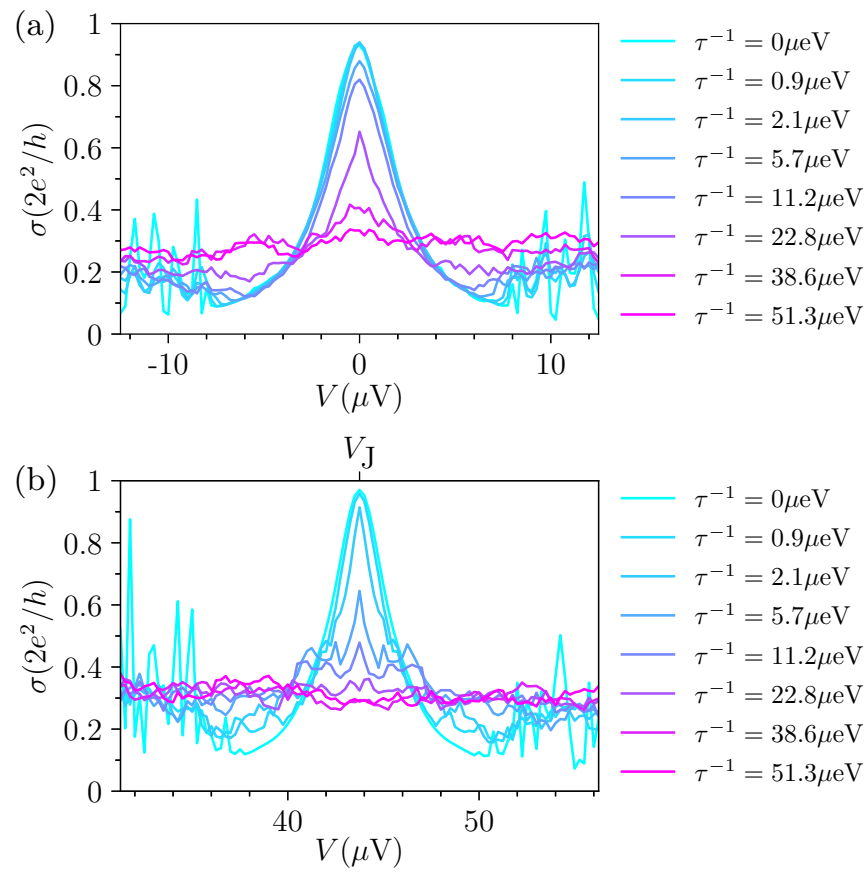

FIG. 4. Conductance as a function of the lead's voltage $V$ for a fixed junction's length, $L_{x}=16 \mu \mathrm{m}$, and different values of disorder strength represented by inverse mean-free time. System parameters are the same as in Figs. 2(a) and 2(b). In (a) we focus on voltages around $V=0$, and in (b) we focus on voltages around $V=V_{\mathrm{J}}$, corresponding to zero and $\pi$ Majorana states, respectively.

where the asymptotic splitting of the Majorana modes is exponentially decaying. The behavior observed here is most likely due to photon-induced coupling between the Majorana states at the two ends of the junction. In this process, a photon can excite a quasiparticle into a conducting mode of the system, allowing for cross talk between the Majorana end states. In Sec. IV we analyze this splitting and show that it becomes small whenever $\left|k_{F} \xi\right| \gg 1$ or when $|\Omega| \gg|\mu|$, where $k_{\mathrm{F}}$ is the Fermi momentum and $\xi$ is the Majorana localization length in the static case.

We end the section on numerical results by demonstrating the robustness of the signatures observed above to weak disorder. We focus on the system parameters used in Figs. 2(a) and 2(b) and simulate random short-correlated disorder, $U(\boldsymbol{r}) U\left(\boldsymbol{r}^{\prime}\right)=\delta\left(\boldsymbol{r}-\boldsymbol{r}^{\prime}\right) /\left(m_{\mathrm{e}} \tau\right)$. Here, $\tau$ is the disorder-induced mean-free time in the case of an unproximitized 2DEG and in the absence of a magnetic field. In Fig. 4, we present results for the conductance as a function of the lead's voltage for different values of $\tau$. Each data point is a result of averaging over 50 disorder realizations. Figures 4(a) and 4(b) focus on voltages near $V=0$ and $V=V_{\mathrm{J}}$, corresponding to zero and $\pi$ Majorana states, respectively. In both cases the Majorana-induced (nearly quantized) peak remains intact for a finite range of disorder strengths, beyond which the peak value begins to decrease until disappearing completely.

Notice the critical value of the disorder corresponds to $\tau^{-1}$, which is of the order of the induced gap in the system, as observed in Figs. 2(a) and 2(b), resembling the behavior of the static topological superconductor in the presence of disorder [50-57]. The difference between static and driven 
topological phases is that the driven system in the present case is, strictly speaking, not gapped (see discussions in Sec. IV). The photon-mediated splitting of the Majorana bound states is suppressed by momentum mismatch, rather than by a gap. In the presence of disorder, however, this momentum mismatch is mediated by impurity scattering. As a result of these processes, whereby single electrons traverse the system (in the $x$ direction), the two Majorana bound states at each end can interact and gap away from zero energy.

\section{PHOTON-INDUCED COUPLING OF THE MAJORANA MODES}

We saw above that while the signatures of the Majorana states are robust, there is a small splitting of the Majorana resonance that does not decay with increasing the system size, as apparent in Fig. 3. Such a splitting does not exist in a static topological superconductor and is a consequence of the periodic drive induced by the voltage bias across the junction.

To analyze this effect we consider a simplified version of the system. We treat the region inside the junction as a 1D semiconductor weakly coupled to the superconductors [see Fig. 1(a)]. For adequate values of the chemical potential and magnetic field, this system is known to be described by a spinless $p$-wave superconductor [34]. Since in our case a voltage bias is applied between the superconductors, the Hamiltonian for the system reads

$$
H_{p}(t)=\sum_{k} \xi_{k} c_{k} c_{k}^{\dagger}+\frac{1}{2}\left[\left(1+e^{-i \Omega t}\right) \Delta_{k} c_{k}^{\dagger} c_{-k}^{\dagger}+\text { H.c. }\right],
$$

where $\Omega=2 e V_{\mathrm{J}}$ as before, $\xi_{k}$ is the dispersion of the lowest electronic band, and $\Delta_{k}=-\Delta_{-k}$ is the effective $p$-wave pairing potential. For concreteness, we take $\Delta(k)=\Delta^{\prime} k$ and $\xi_{k}=k^{2} / 2 m-\bar{\mu}$ for some effective values of the electron mass $m$, the chemical potential $\bar{\mu}$, and the coefficient $\Delta^{\prime}$. We emphasize, however, that in the weak-pairing limit, the physics is determined by $\Delta_{k}$ and $\xi_{k}$ near the Fermi momentum $k_{\mathrm{F}}$, defined by $\xi_{k_{\mathrm{F}}}=0$. We assume this limit below.

In the absence of the time-dependent term, the Hamiltonian of Eq. (5) has a gap at the Fermi level $(\varepsilon=0)$. The periodictime-dependent term, however, enables a process in which, by absorbing a photon, a quasiparticle can be excited into a conducting mode. Focusing on the zero Majorana, we retain only the $n=0$ Floquet sector and Floquet bands which can be reached by absorbing at most a single photon to leading order in $\left|\Delta\left(k_{\mathrm{F}}\right)\right| / \Omega$. The resulting Hamiltonian can be written in first-quantization form as

$\mathcal{H}_{p}^{\mathrm{F}}=\frac{1+\lambda_{z}}{2}\left(\xi_{k} \tau_{z}+\Delta^{\prime} k \tau_{x}\right)+\frac{1-\lambda_{z}}{2}\left(\xi_{k}-\Omega\right) \tau_{z}+\Delta^{\prime} k \lambda_{x} \tau_{x}$,

where $\lambda_{x, y, z}$ are Pauli matrices operating on the space of states having $\{0,1\}$ photons. The first term above corresponds to the zero-photon sector and describes a static spinless $p$-wave superconductor. The second term corresponds to the one-photon sector and describes a gapless 1D channel. Finally, the third term couples the two sectors and describes electron pairing mediated by an absorption or emission of a photon. We note that a similarly structured Hamiltonian can be obtained for describing the $\pi$ Majorana by focusing on quasienergies near $\varepsilon=\Omega / 2$ instead of $\varepsilon=0$.

To make analytic progress, we first treat the zero-photon sector by solving for the Majorana end states, $\gamma_{L}$ and $\gamma_{R}$, that emerge in the presence of open-boundary conditions and projecting out the rest of the spectrum. We then integrate out the one-photon modes to obtain a self-energy term describing a coupling between the two Majorana end states, in addition to the exponentially small finite-size coupling. The result for the $2 \times 2$ Green's function of the ground-state manifold, written in the Nambu basis $\left(\gamma_{\mathrm{L}}, \gamma_{\mathrm{R}}\right)$, is given by

$$
G^{\mathrm{R}}(\omega)=\left[\omega-\epsilon_{\mathrm{M}} \tau_{y}-\Sigma(\omega)\right]^{-1},
$$

where $\Sigma(\omega)$ is the self-energy due to photon-mediated pairing and $\epsilon_{\mathrm{M}} \propto \exp (-L / \xi)$ is the exponentially decaying energy splitting between the Majorana states in the static case, with the decay length given by $\xi=1 /\left(m\left|\Delta^{\prime}\right|\right)$. We note that in the weak-pairing limit one has $k_{\mathrm{F}} \xi \gg 1$.

In the limit of $L \gg \xi$, one can neglect $\epsilon_{M}$. The shift and broadening of the Majorana resonance can then be obtained from the zero-frequency self-energy, which to leading order in $1 /\left(k_{\mathrm{F}} \xi\right)$ reads

$$
\Sigma(0)=\frac{\Delta^{\prime} k_{\mathrm{F}}}{\left(k_{\mathrm{F}} \xi\right)^{2}}\left(\frac{\bar{\mu}}{\Omega}\right)^{2}\left[\frac{32}{k_{\mathrm{F}} \xi}\left(1+\frac{\bar{\mu}}{\Omega}\right) \tau_{y}-i 8 \sqrt{1+\frac{\Omega}{\bar{\mu}}}\right] .
$$

The first term in Eq. (8) gives the energy splitting of the Majorana modes, while the second term gives its broadening and represents the hybridization of the Majorana state with the continuum of extended modes in the wire. Considering the self-energy for finite values of $\omega$ results in corrections to the splitting and the broadening; however, we have verified that these involve a higher order of $1 /\left(k_{\mathrm{F}} \xi\right)$.

From Eq. (8) it is evident that, unlike in the static case, the splitting between the Majorana states does not decay with the length of the system. Nevertheless, in the limit of either $k_{F} \xi \gg 1$ or $\Omega \gg \bar{\mu}$, this splitting can be much smaller than the induced superconducting gap in the system $\Delta^{\prime} k_{\mathrm{F}}$. Such a situation is, indeed, observed in the numerical simulations of Sec. III, which is apparent from Fig. 3. For short enough system lengths, $\epsilon_{\mathrm{M}}$ dominates over $\Sigma(0)$ in Eq. (7), and the splitting of the Majorana modes follows oscillations with exponentially decaying amplitude. For a longer system size, $\Sigma(0)$ dominates over $\epsilon_{\mathrm{M}}$, and the splitting between the Majorana modes follows a constant value.

We can compare the results with the numerical simulations of Sec. III. We can extract from the numerical simulations that $\Delta_{\text {ind }}=\Delta^{\prime} k_{\mathrm{F}} \approx 8 \mu \mathrm{eV}, k_{\mathrm{F}} \xi \approx 20$, and $\Omega / \bar{\mu} \approx 0.5$. Inserting these values into Eq. (8), we obtain the splitting and the width of the resonance, $\Sigma(0) \approx 0.38 \tau_{y}-0.78 i(\mu \mathrm{eV})$. This splitting is consistent with the one observed in Fig. 3. The width obtained in Eq. (8) should be understood as an average over the finite-size oscillation observed in Fig. 3.

Note that while the broadening in Eq. (8) is parametrically larger in $1 / k_{F} \xi$ than the splitting, for our parameters the larger numerical prefactor of the splitting is sufficient to lead to similar values for splitting and broadening. In general, there will be other contributions to the self-energy, $\Sigma(0) \rightarrow \Sigma(0)+\tilde{\Sigma}$, that go beyond the continuum modes, and the splitting is just 
observable for sufficiently small $\tilde{\Sigma}$. One example, mentioned above, is the broadening by the coupling to the transport leads. Also, interactions and phonon-induced relaxation will contribute to $\tilde{\Sigma}$. We note that when a decay rate $\gamma$ is included in the Green's function in Eq. (7), this contribution is $\tilde{\Sigma}=$ $2 i \gamma\left(\Delta^{\prime} k_{F}\right)^{2} / \Omega^{2}$, which can be neglected relative to the broadening of Eq. (8) for $\gamma \ll \Delta^{\prime} k_{F} \sqrt{1+\Omega / \bar{\mu}}=\Delta_{\text {ind }} \sqrt{1+\Omega / \bar{\mu}}$. Typical phonon relaxation times correspond to $\gamma \lesssim 1 \mu \mathrm{eV}$ and can therefore be neglected.

\section{DISCUSSION}

We have investigated a voltage-biased Josephson junction implemented in a two-dimensional electron gas in the presence of an in-plane magnetic field. We have shown that this system supports a pair of weakly coupled zero Majorana end states together with a pair of weakly coupled $\pi$ Majorana states. The weak coupling between Majorana end states on opposite sides of the junction is induced by photon absorption or emission, which causes the Majorana modes to hybridize with the highly excited conducting modes. As we show, this coupling can, nevertheless, become exceedingly small for reasonable system parameters.

For a phase-biased Josephson junction of the type we study here, it was previously shown that the system supports zeroenergy Majorana bound states at each end of the junction $[23,24]$. Such a system was subsequently studied by several experimental groups who observed signatures of topological superconductivity [26-28]. Our results suggest that a slight modification of the same experimental setup can realize a Floquet topological superconductor. The presence of zero Majorana and $\pi$ Majorana states in such a system can be directly probed by measuring DC differential conductance from a metallic lead coupled to one of the junction's ends as a function of its voltage $V$, as depicted in Fig. 1(a). This should produce simultaneous nearly quantized resonances at $V=0$ and $V=V_{\mathrm{J}}$, respectively, with the latter being the voltage bias across the junction (see Fig. 2).

Further insight into the origin of these resonances can be gained by considering a situation where the system is physically split into two parts at the middle of the junction $(y=0)$. Each subsystem then consists of a superconductor in proximity to a 1D semiconductor and can therefore be tuned into a (static) topological superconducting phase [58,59], giving rise to a Majorana bound state at each of its ends. Since the Fermi energies of the two subsystems differ by $V_{\mathrm{J}}$, one pair of Majorana bound states resides at energy $\varepsilon=0$, while the other resides at $\varepsilon=V_{\mathrm{J}}$. These two pairs of Majorana bound states result in conductance peaks at $V=0$ and $V_{\mathrm{J}}$, respectively. Interestingly, these features survive even when the two subsystems are brought together, as shown in this work. Indeed, the voltage-biased Josephson junction allows electrons (and holes) to gain energy through multiple Andreev reflections and escape the gap to a conducting channel, possibly hybridizing Majorana states at opposite ends. As shown in Secs. III and IV, however, in practice this hybridization is rather weak.

Applying a voltage bias to the junction in search of a Floquet topological superconductor has the added advantage of introducing a tuning parameter into the system, $V_{\mathrm{J}}$, in addition to the junction chemical potential $\mu$ (controlled by a gate) and the in-plane magnetic field. In the weak-pairing limit, zero $(\pi)$ Majorana modes should appear whenever $\mu\left(V_{\mathrm{J}}\right)$ crosses through an odd number of Zeeman-split bands [see Fig. 1(b)]. This was demonstrated numerically (see Fig. 2), together with the robustness of the Majorana modes to disorder (see Fig. 4).

An exciting prospect of Floquet topological superconductors is the ability to implement braiding of Majorana modes in a strictly 1D system [7-9]. In this scenario one takes advantage of the fact that the zero and $\pi$ Majoranas can be thought of as residing in separate channels. Such a process can, in principle, be implemented in the system considered here by adding local gates to control the position of the Majorana modes, together with an additional AC potential to couple the zero and $\pi$ Majorana modes in restricted regions. The photon-mediated coupling between opposite Majorana end states discussed above will, in principle, cause the braiding operation to be unprotected, as it can induce a nonuniversal dynamical phase. Nevertheless, one might be able to avoid this by performing the braiding on a timescale shorter than the inverse energy splitting of the Majoranas.

\section{ACKNOWLEDGMENTS}

This research was supported by the Institute of Quantum Information and Matter, an NSF Frontier Center funded by the Gordon and Betty Moore Foundation, the Packard Foundation, and the Simons Foundation. A.H. acknowledges support from the Walter Burke Institute for Theoretical Physics at the California Institute of Technology. The results of this work were obtained prior to the employment of A.H. at the Amazon Web Services Center for Quantum Computing. G.R. is also grateful for support through NSF DMR Grant No. 1839271. This work was performed in part at the Aspen Center for Physics, which is supported by National Science Foundation Grant No. PHY-1607611.

\section{APPENDIX A: NUMERICAL RESULTS FOR A NARROW JUNCTION}

Decreasing the width of the junction $w$ is expected to increase the induced superconducting gap in the junction. Decreasing $w$ also causes higher transverse modes to get pushed to high energies. In this case, the relevant scenario for observing simultaneous zero and $\pi$ Majorana modes is when the lines $\varepsilon=0$ and $\varepsilon=\Omega / 2=e V_{\mathrm{J}}$ both cross a single pair of Fermi points [see scenario (i) in Fig. 1(b)]. The larger splitting between transverse modes allows one to increase the Zeeman field and $\Omega$, thereby suppressing photon-induced coupling of the Majorana modes to the bulk conducting mode. In Fig. 5 we present numerical results for the differential conductance as a function of voltage and system length for $w=$ $73 \mathrm{~nm}, \mu_{\mathrm{J}}=287.5 \mu \mathrm{eV}, \mu_{\mathrm{SC}}=1.125 \mathrm{meV}, E_{\mathrm{Z}}^{0}=250 \mu \mathrm{eV}$, and $V_{\mathrm{J}}=125 \mu \mathrm{V}$, keeping the rest of the system parameters unchanged. The conductance spectrum exhibits simultaneous resonances at $V=0$ and $V=V_{\mathrm{J}}$, each separated by a sizable gap of about $40 \mu \mathrm{eV}$.

\section{APPENDIX B: LINECUTS OF CONDUCTANCE SPECTRA}

To facilitate easier comparison of the results presented in Fig. 2 we append here linecuts showing the conductance as 


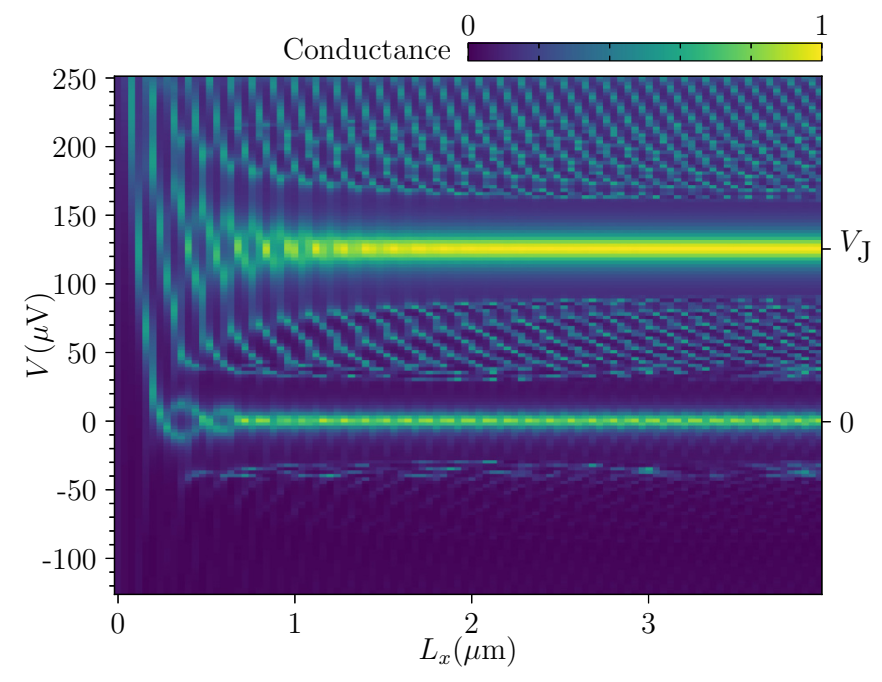

FIG. 5. Conductance as a function of the lead's voltage $V$ and the junction's length $L_{x}$, in units of $2 e^{2} / h$. The voltage across the junction is taken to be $V_{\mathrm{J}}=125 \mu \mathrm{V}$.

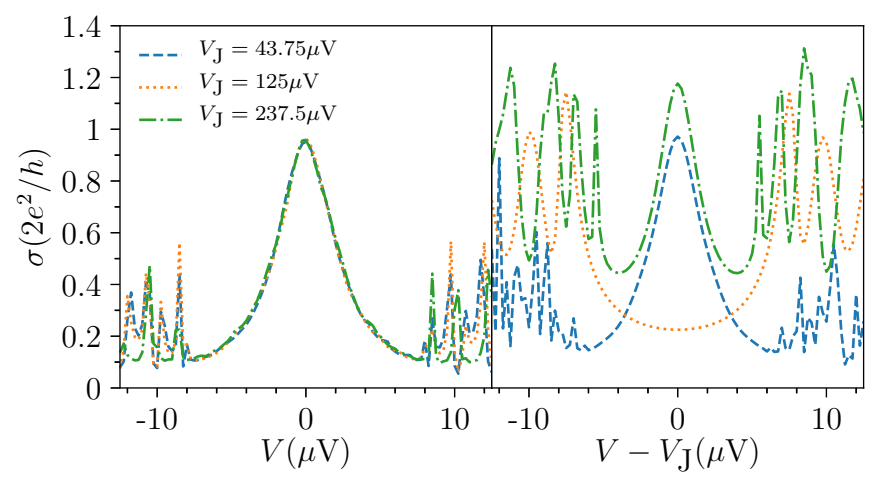

FIG. 6. Linecuts from Fig. 2, showing conductance versus voltage for a fixed system length of $L_{x}=16 \mu \mathrm{m}$. Left: Zero Majorana. Right: $\pi$ Majorana.

a function of voltage for a fixed system length. They are presented in Fig. 6.
[1] L. Jiang, T. Kitagawa, J. Alicea, A. R. Akhmerov, D. Pekker, G. Refael, J. I. Cirac, E. Demler, M. D. Lukin, and P. Zoller, Majorana Fermions in Equilibrium and in Driven Cold-Atom Quantum Wires, Phys. Rev. Lett. 106, 220402 (2011).

[2] V. Khemani, A. Lazarides, R. Moessner, and S. L. Sondhi, Phase Structure of Driven Quantum Systems, Phys. Rev. Lett. 116, 250401 (2016).

[3] D. V. Else, B. Bauer, and C. Nayak, Floquet Time Crystals, Phys. Rev. Lett. 117, 090402 (2016).

[4] C. W. von Keyserlingk, V. Khemani, and S. L. Sondhi, Absolute stability and spatiotemporal long-range order in Floquet systems, Phys. Rev. B 94, 085112 (2016).

[5] N. Y. Yao, A. C. Potter, I.-D. Potirniche, and A. Vishwanath, Discrete Time Crystals: Rigidity, Criticality, and Realizations, Phys. Rev. Lett. 118, 030401 (2017).

[6] J. Zhang, P. W. Hess, A. Kyprianidis, P. Becker, A. Lee, J. Smith, G. Pagano, I.-D. Potirniche, A. C. Potter, A. Vishwanath, N. Y. Yao, and C. Monroe, Observation of a discrete time crystal, Nature (London) 543, 217 (2017).

[7] R. W. Bomantara and J. Gong, Simulation of Non-Abelian Braiding in Majorana Time Crystals, Phys. Rev. Lett. 120, 230405 (2018).

[8] R. W. Bomantara and J. Gong, Quantum computation via Floquet topological edge modes, Phys. Rev. B 98, 165421 (2018).

[9] B. Bauer, T. Pereg-Barnea, T. Karzig, M.-T. Rieder, G. Refael, E. Berg, and Y. Oreg, Topologically protected braiding in a single wire using Floquet Majorana modes, Phys. Rev. B 100, 041102(R) (2019).

[10] L. Yuan, Y. Shi, and S. Fan, Photonic gauge potential in a system with a synthetic frequency dimension, Opt. Lett. 41, 741 (2016).

[11] T. Ozawa, H. M. Price, N. Goldman, O. Zilberberg, and I. Carusotto, Synthetic dimensions in integrated photonics: From optical isolation to four-dimensional quantum Hall physics, Phys. Rev. A 93, 043827 (2016).
[12] I. Martin, G. Refael, and B. Halperin, Topological Frequency Conversion in Strongly Driven Quantum Systems, Phys. Rev. X 7, 041008 (2017).

[13] Y. Peng and G. Refael, Time-quasiperiodic topological superconductors with Majorana multiplexing, Phys. Rev. B 98, 220509(R) (2018).

[14] D. T. Liu, J. Shabani, and A. Mitra, Floquet Majorana zero and $\pi$ modes in planar Josephson junctions, Phys. Rev. B 99, 094303 (2019).

[15] Y. Li, A. Kundu, F. Zhong, and B. Seradjeh, Tunable Floquet Majorana fermions in driven coupled quantum dots, Phys. Rev. B 90, 121401(R) (2014).

[16] A. A. Reynoso and D. Frustaglia, Unpaired Floquet Majorana fermions without magnetic fields, Phys. Rev. B 87, 115420 (2013).

[17] M. Thakurathi, A. A. Patel, D. Sen, and A. Dutta, Floquet generation of Majorana end modes and topological invariants, Phys. Rev. B 88, 155133 (2013).

[18] M. Thakurathi, D. Loss, and J. Klinovaja, Floquet Majorana fermions and parafermions in driven Rashba nanowires, Phys. Rev. B 95, 155407 (2017).

[19] D. J. Yates and A. Mitra, Entanglement properties of the timeperiodic Kitaev chain, Phys. Rev. B 96, 115108 (2017).

[20] D. Yates, Y. Lemonik, and A. Mitra, Central Charge of Periodically Driven Critical Kitaev Chains, Phys. Rev. Lett. 121, 076802 (2018).

[21] N. Müller, D. M. Kennes, J. Klinovaja, D. Loss, and H. Schoeller, Electronic transport in one-dimensional Floquet topological insulators via topological and nontopological edge states, Phys. Rev. B 101, 155417 (2020).

[22] M. Hell, M. Leijnse, and K. Flensberg, Two-Dimensional Platform for Networks of Majorana Bound States, Phys. Rev. Lett. 118, 107701 (2017).

[23] F. Pientka, A. Keselman, E. Berg, A. Yacoby, A. Stern, and B. I. Halperin, Topological Superconductivity in a Planar Josephson Junction, Phys. Rev. X 7, 021032 (2017). 
[24] M. Hell, K. Flensberg, and M. Leijnse, Coupling and braiding Majorana bound states in networks defined in two-dimensional electron gases with proximity-induced superconductivity, Phys. Rev. B 96, 035444 (2017).

[25] D. T. Liu, J. Shabani, and A. Mitra, Long-range Kitaev chains via planar Josephson junctions, Phys. Rev. B 97, 235114 (2018).

[26] H. Ren, F. Pientka, S. Hart, A. T. Pierce, M. Kosowsky, L. Lunczer, R. Schlereth, B. Scharf, E. M. Hankiewicz, L. W. Molenkamp, B. I. Halperin, and A. Yacoby, Topological superconductivity in a phase-controlled Josephson junction, Nature (London) 569, 93 (2019).

[27] M. C. Dartiailh, W. Mayer, J. Yuan, K. S. Wickramasinghe, A. Matos-Abiague, I. Žutić, and J. Shabani, Phase Signature of Topological Transition in Josephson Junctions, Phys. Rev. Lett. 126, 036802 (2021).

[28] A. Fornieri, A. M. Whiticar, F. Setiawan, E. Portolés, A. C. C. Drachmann, A. Keselman, S. Gronin, C. Thomas, T. Wang, R. Kallaher, G. C. Gardner, E. Berg, M. J. Manfra, A. Stern, C. M. Marcus, and F. Nichele, Evidence of topological superconductivity in planar Josephson junctions, Nature (London) 569, 89 (2019).

[29] A. Haim and A. Stern, Benefits of Weak Disorder in OneDimensional Topological Superconductors, Phys. Rev. Lett. 122, 126801 (2019).

[30] F. Setiawan, A. Stern, and E. Berg, Topological superconductivity in planar Josephson junctions: Narrowing down to the nanowire limit, Phys. Rev. B 99, 220506(R) (2019).

[31] B. Scharf, F. Pientka, H. Ren, A. Yacoby, and E. M. Hankiewicz, Tuning topological superconductivity in phasecontrolled Josephson junctions with Rashba and Dresselhaus spin-orbit coupling, Phys. Rev. B 99, 214503 (2019).

[32] S. Ikegaya, S. Tamura, D. Manske, and Y. Tanaka, Anomalous proximity effect of planar topological Josephson junctions, Phys. Rev. B 102, 140505(R) (2020).

[33] A. Kitaev, Unpaired Majorana fermions in quantum wires, Phys. Usp. 44, 131 (2001).

[34] J. Alicea, New directions in the pursuit of Majorana fermions in solid state systems., Rep. Prog. Phys. 75, 076501 (2012).

[35] C. W. J. Beenakker, Search for Majorana Fermions in Superconductors, Annu. Rev. Condens. Matter Phys. 4, 113 (2013).

[36] R. Aguado, Majorana quasiparticles in condensed matter, Riv. Nuovo Cimento, 40, 523 (2017).

[37] R. Lutchyn, E. Bakkers, L. Kouwenhoven, P. Krogstrup, C. Marcus, and Y. Oreg, Majorana zero modes in superconductorsemiconductor heterostructures, Nat. Rev. Mater. 3, 52 (2018).

[38] P. A. Lee and D. S. Fisher, Anderson Localization in Two Dimensions, Phys. Rev. Lett. 47, 882 (1981).

[39] A. Haim, R. Ilan, and J. Alicea, Quantum Anomalous Parity Hall Effect in Magnetically Disordered Topological Insulator Films, Phys. Rev. Lett. 123, 046801 (2019).

[40] G. E. Blonder, M. Tinkham, and T. M. Klapwijk, Transition from metallic to tunneling regimes in superconducting microconstrictions: Excess current, charge imbalance, and supercurrent conversion, Phys. Rev. B 25, 4515 (1982).

[41] M. Moskalets and M. Büttiker, Floquet scattering theory of quantum pumps, Phys. Rev. B 66, 205320 (2002).
[42] M. V. Moskalets, Scattering Matrix Approach to Non-stationary Quantum Transport (World Scientific, London, 2012).

[43] A. Kundu and B. Seradjeh, Transport Signatures of Floquet Majorana Fermions in Driven Topological Superconductors, Phys. Rev. Lett. 111, 136402 (2013).

[44] C. J. Bolech and E. Demler, Observing Majorana Bound States in $p$-Wave Superconductors Using Noise Measurements in Tunneling Experiments, Phys. Rev. Lett. 98, 237002 (2007).

[45] K. T. Law, P. A. Lee, and T. K. Ng, Majorana Fermion Induced Resonant Andreev Reflection, Phys. Rev. Lett. 103, 237001 (2009).

[46] K. Flensberg, Tunneling characteristics of a chain of Majorana bound states, Phys. Rev. B 82, 180516(R) (2010).

[47] L. Fidkowski, J. Alicea, N. H. Lindner, R. M. Lutchyn, and M. P. A. Fisher, Universal transport signatures of Majorana fermions in superconductor-Luttinger liquid junctions, Phys. Rev. B 85, 245121 (2012).

[48] C. W. J. Beenakker, J. P. Dahlhaus, M. Wimmer, and A. R. Akhmerov, Random-matrix theory of Andreev reflection from a topological superconductor, Phys. Rev. B 83, 085413 (2011).

[49] A. Farrell and T. Pereg-Barnea, Edge-state transport in Floquet topological insulators, Phys. Rev. B 93, 045121 (2016).

[50] O. Motrunich, K. Damle, and D. A. Huse, Griffiths effects and quantum critical points in dirty superconductors without spinrotation invariance: One-dimensional examples, Phys. Rev. B 63, 224204 (2001).

[51] P. W. Brouwer, M. Duckheim, A. Romito, and F. von Oppen, Probability Distribution of Majorana End-State Energies in Disordered Wires, Phys. Rev. Lett. 107, 196804 (2011).

[52] A. M. Lobos, R. M. Lutchyn, and S. Das Sarma, Interplay of Disorder and Interaction in Majorana Quantum Wires, Phys. Rev. Lett. 109, 146403 (2012).

[53] F. Pientka, A. Romito, M. Duckheim, Y. Oreg, and F. von Oppen, Signatures of topological phase transitions in mesoscopic superconducting rings, New J. Phys. 15, 025001 (2013).

[54] D. A. Huse, R. Nandkishore, V. Oganesyan, A. Pal, and S. L. Sondhi, Localization-protected quantum order, Phys. Rev. B 88, 014206 (2013).

[55] M.-T. Rieder, P. W. Brouwer, and I. Adagideli, Reentrant topological phase transitions in a disordered spinless superconducting wire, Phys. Rev. B 88, 060509(R) (2013).

[56] I. Adagideli, M. Wimmer, and A. Teker, Effects of electron scattering on the topological properties of nanowires: Majorana fermions from disorder and superlattices, Phys. Rev. B 89, 144506 (2014).

[57] M.-T. Rieder and P. W. Brouwer, Density of states at disorderinduced phase transitions in a multichannel majorana wire, Phys. Rev. B 90, 205404 (2014).

[58] Y. Oreg, G. Refael, and F. von Oppen, Helical Liquids and Majorana Bound States in Quantum Wires, Phys. Rev. Lett. 105, 177002 (2010).

[59] R. M. Lutchyn, J. D. Sau, and S. Das Sarma, Majorana Fermions and a Topological Phase Transition in Semiconductor-Superconductor Heterostructures, Phys. Rev. Lett. 105, 077001 (2010). 